\title{
Abundances and possible diffusion of elements in M 67 stars ${ }^{\star}$
}

\author{
Anna Önehag ${ }^{1}$, Bengt Gustafsson ${ }^{1,2}$, and Andreas Korn ${ }^{1}$ \\ 1 Department of Physics and Astronomy, Uppsala Astronomical Observatory, Box 515, 75120 Uppsala, Sweden \\ e-mail: Anna. Onehag@physics.uu.se \\ 2 Nordita, Roslagstullsbacken 23, 10691 Stockholm, Sweden
}

Received 13 September 2013 / Accepted 25 November 2013

\section{ABSTRACT}

\begin{abstract}
Context. The rich open cluster M 67 is known to have a chemical composition close to solar and an age of about 3.5-4.8 Gyr. It offers an important opportunity to check and develop our understanding of the physics and the evolution of solar-type stars.

Aims. We present a spectroscopic study at high resolution, $R \approx 50000$, of 14 stars located on the main sequence, at the turn-off point, and on the early subgiant branch in the cluster in order to investigate its detailed chemical composition, for comparison with the Sun and solar twins in the solar neighbourhood, and to explore selective atomic diffusion of chemical elements as predicted by stellar-structure theory.

Methods. We have obtained VLT/FLAMES-UVES spectra and analysed these strictly differentially in order to explore chemicalabundance similarities and differences between the M 67 stars and the Sun and among the M 67 stars themselves.

Results. Individual abundances of 19 different chemical elements are obtained for the stars. They are found to agree very well with solar abundances, with abundance ratios closer to solar than those of most solar twins in the solar neighbourhood. An exception is $\mathrm{Li}$, which shows considerable scatter among the cluster stars. There is a tendency for the cluster-star abundances to be more depleted than the abundances in the field stars in correlation with the condensation temperature of the elements, a tendency also found earlier for the Sun. Moreover, the heavy-element abundances are found to be reduced in the hotter stars and dwarfs by typically $\leq 0.05$ dex, as compared to the abundances of the subgiants.

Conclusions. The results support the hypothesis that the gas of the proto-cluster was depleted by formation and cleansing of dust before the stars formed. They also add support to the proposal that the Sun was formed in a dense stellar environment. Moreover, the observed minor reductions of heavy elements, relative to our standard star M 67-1194 and the subgiants, in the atmospheres of dwarfs and turn-off point stars seem to suggest that diffusion processes are at work in these stars, although the evidence is not compelling. Based on theoretical models, the diffusion-corrected initial metallicity of M 67 is estimated to be $[\mathrm{Fe} / \mathrm{H}]=+0.06$.
\end{abstract}

Key words. stars: atmospheres - stars: fundamental parameters - stars: abundances - techniques: spectroscopic open clusters and associations: individual: M 67 - methods: observational

\section{Introduction}

The old and rich open cluster M 67 offers interesting possibilities for studying the evolution of solar-like stars. The cluster has a solar-similar chemical composition with $[\mathrm{Fe} / \mathrm{H}]$ in the range -0.04 to +0.03 (Hobbs \& Thorburn 1991; Tautvaišiene et al. 2000; Yong et al. 2005; Randich et al. 2006; Pace et al. 2008; Pasquini et al. 2008). Its age is also comparable to the Sun: $3.5-4.8 \mathrm{Gyr}$ (Yadav et al. 2008). M 67 is relatively nearby ( 800-900 pc, Majaess et al. 2011; Sarajedini et al. 2009; Yakut et al. 2009) and is affected only a little by interstellar extinction, which allows for detailed spectroscopic studies of even its mainsequence stars.

M 67 thus seems to offer good possibilities of finding solartwin candidates for further exploration. Pasquini et al. (2008) (followed by a paper of Biazzo et al. 2009) have listed ten promising twin candidates in the cluster. We have previously analysed one of these spectroscopically (M 67-1194, Önehag et al. 2011) and found it to have a chemical composition very close to solar. This is noteworthy since Meléndez et al. (2009) and Ramírez et al. (2009) have systematically analysed solartwin candidates in the solar neighbourhood and found that,

\footnotetext{
* Appendix A is available in electronic form at http://www. aanda.org
}

although these stars in general have fundamental parameters very close to solar, differential high-accuracy abundance analyses prove that almost all of them have chemical compositions systematically deviating from that of the Sun: the twins are, in relative terms, slightly richer in refractory elements (that condense at high temperatures) than volatiles. Unlike these nearby solar twins, the composition of M 67-1194 is more solar-like, which lends some support to the idea that the Sun was once formed in a similar cluster environment. It is of strong interest to investigate whether analyses of other stars in M 67 verify this remarkable solar likeness.

A cluster like M 67 also offers the possibility of exploring the changes in surface composition of solar-type stars as a result of selective diffusion of elements in the stars. Such effects are expected to deplete heavy elements from the surface, but to different degrees for different elements depending on the element-specific radiative levitation. Effects of this nature have been found by Korn et al. (2007), Lind et al. (2008), and Nordlander et al. (2012) for the metal-poor globular cluster NGC 6397. These authors compared the abundances of turnoff point stars, subgiants, and red giants, where the cooler stars are mixed by the deeper convection zone and thus are less affected, and found satisfactory agreement with theoretical predictions of atomic diffusion moderated by surface convection 
and (parametrised) additional mixing of unknown origin. Similar effects, albeit of smaller overall amplitude, have recently been traced in NGC 6752 at somewhat higher metallicities (Gruyters et al. 2013). In M 4, at roughly one tenth solar metallicity, no abundance trend in iron was found between the turn-off point and the red giants (Mucciarelli et al. 2011), possibly indicating that the more massive outer convection zones of more metal-rich stars suppress atomic diffusion.

It is now important to find out to what extent such diffusion effects are also visible in dwarf stars of solar metallicity. This is particularly important since solar-like stars are used as the main tracers of the chemical evolution in the Galaxy, and any physical effects that change their surface elemental composition during their evolution must then be taken into consideration in detailed work on Galactic evolution.

Here we present the analysis of 14 stars in M 67, six mainsequence (MS) stars (including M 67-1194, which is used as a standard star in this study), three stars at the turn-off point (TO), and five on the early subgiant branch (SG). The analysis is based on high-resolution observations with relatively high signal-tonoise ratio $(\mathrm{S} / \mathrm{N})$. In Sect. 2, we present the observations and the data reduction. Section 3 describes the analysis method and the estimation of fundamental parameters $\left(T_{\mathrm{eff}}, \log g,[\mathrm{Fe} / \mathrm{H}]\right.$, and $\xi_{\mathrm{t}}$ ). In Sect. 4 we present the results of a detailed analysis of a number of chemical elements and discuss the results. In Sect. 5 we summarise our main results.

\section{Observations}

The observations of M67-1194 were carried out with the multi-object spectrograph FLAMES/UVES at ESO-VLT UT2 in Service Mode in the spring of 2009 during a period of three months (18th of January-3rd of April, project 082.D-0726(A), P. I. Gustafsson). The observations were arranged such that approximately a similar number of photons were collected for each star, resulting in longer total exposure times for the fainter stars. In each observing block of the observations, one fibre of the spectrograph system was positioned on M 67-1194 in order to collect as many observations as possible of this faintest star in the programme.

We obtained altogether 23 individual observations in $13 \mathrm{ob}-$ serving nights (18 h of net exposure time). The chosen spectrograph setting (RED580) yields a resolution of $R=\lambda / \Delta \lambda=$ 47000 ( $1^{\prime \prime}$ fibre) and a wavelength coverage of 4800-6700 $\mathrm{A}$. A typical $\mathrm{S} / \mathrm{N}$ per frame of 50 per unbinned pixel was achieved. Baryocentric radial velocities were determined from the individual spectra indicating a radial velocity of $33 \pm 1 \mathrm{~km} \mathrm{~s}^{-1}$. This is in excellent agreement with the mean radial velocity of the cluster as determined by Yadav et al. (2008), giving $33.67 \pm 0.09 \mathrm{~km} \mathrm{~s}^{-1}$. The frames were subsequently co-added for highest possible $\mathrm{S} / \mathrm{N}$.

\subsection{Data reduction}

While ESO provides pipeline-reduced data for an initial assessment of the observed spectra, the resulting data are not intended for a full scientific analysis. In fact, we found that the extraction of our spectra by the ESO pipeline was problematic, mainly because the instrumental setup of FLAMES/UVES combined with our choice of targets is challenging. Having eight fibres tightly packed in a slit-like fashion, FLAMES/UVES can obtain echelle spectra of up to 8 targets in parallel. However, the spacing between the individual fibres is rather small, which leads to non-negligible light contamination between adjacent fibres when the apparent magnitudes of the targets differ significantly. This was the case, as M67-1194 is considerably fainter than any of the other stars placed on one of the adjacent fibres. The ESO pipeline had severe problems extracting such a spectrum, which is apparent from a resulting strong and periodic pattern of lowsensitivity spikes. These difficulties were overcome using a special reduction procedure, based on the echelle-reduction package REDUCE (Piskunov \& Valenti 2002), and further described in Önehag et al. (2011). In this work we benefited from important contributions by Dr. Eric Stempels. The periodic pattern of spikes was removed successfully, and the agreement with the pipeline-reduced spectra outside the spikes is excellent. The $\mathrm{S} / \mathrm{N}$ in the co-added spectra of the program star is typically 150 per rebinned pixel, where a rebinning by two has been applied retaining the full resolution of FLAMES-UVES.

\section{Analysis}

\subsection{Fundamental stellar parameters}

Effective temperatures: in establishing the effective temperatures of the programme stars we have used the colours $\left(V-K_{\mathrm{s}}\right)$ and $\left(V-I_{\mathrm{c}}\right)$, as well as the wings of the $\mathrm{H} \alpha$ lines, as primary indicators. We preferred the use of colours and $\mathrm{H} \alpha$-line profiles to the use of metal-lines of different excitation and ionisation. The equilibria of atoms and ions are known to be affected by departures from LTE, and since we aimed at exploring small differences in abundances between stars with different surface gravities (i.e. atmospheric pressures) and effective temperatures, we were anxious to avoid systematic errors due to the problems of modelling such non-LTE effects and their pressure and temperature dependencies (in particular the effect of inelastic collisions with neutral hydrogen as well as the exact temperature sensitivity of the photo-ionising ultraviolet flux) from first principles. However, in checking our temperature scale we investigated the temperatures resulting also from the excitation of Fe I and the ionisation of $\mathrm{Fe}$ and $\mathrm{Ti}$, see below.

The $\left(V-K_{\mathrm{s}}\right)$ and $\left(V-I_{\mathrm{c}}\right)$ colours were adopted from the 2MASS Catalogue (Cutri et al. 2003) and Yadav et al. (2008), respectively, with $K_{\mathrm{s}}$ measured in the 2MASS and $I_{\mathrm{c}}$ measured in the Johnson-Cousins system. A reddening $E(B-V)$ of $0.041 \pm 0.004$ (Taylor 2007) was adopted and the $T_{\text {eff }}$ calibrations of Casagrande et al. (2010) applied. In these calibrations neither differences in $\log g$ among the stars at or near the main sequence, nor the effects of varying microturbulence parameters were considered. We verified that these effects only correspond to $\Delta T_{\text {eff }} \sim 30 \mathrm{~K} / \mathrm{dex} \times \Delta \log g$ and $\Delta T_{\text {eff }} \sim-5 \mathrm{~K} / \mathrm{km} \mathrm{s}^{-1} \times \Delta \xi_{\mathrm{t}}$ by model-atmosphere calculations performed with the MARCS program (Gustafsson et al. 2008). In practice, these corrections can thus be neglected.

In fitting the wings of the $\mathrm{H} \alpha$ lines we used the synthetic spectrum programme SME (Valenti \& Piskunov 1996; Valenti $\&$ Fischer 2005) and MARCS models (Gustafsson et al. 2008). SME uses the $\mathrm{H} \alpha$ line-broadening data of Barklem et al. (2000). We started by comparing the wings calculated using a MARCS $T_{\text {eff }} / \log g /[\mathrm{Fe} / \mathrm{H}]=5780 \mathrm{~K} / 4.44 / 0.0$ solar model with the Kitt Peak Atlas (Kurucz et al. 1984) of the observed solar flux. The result is shown in Fig. 1. Obviously, the calculated profile is too wide, and a good solar fit would require a model temperature around $5670 \mathrm{~K}$. Assuming that models for slightly hotter stars would suffer from a similar mismatch to observations, we have schematically increased the temperatures derived from the $\mathrm{H} \alpha$ fits by $100 \mathrm{~K}$. This result agrees very well with that of 


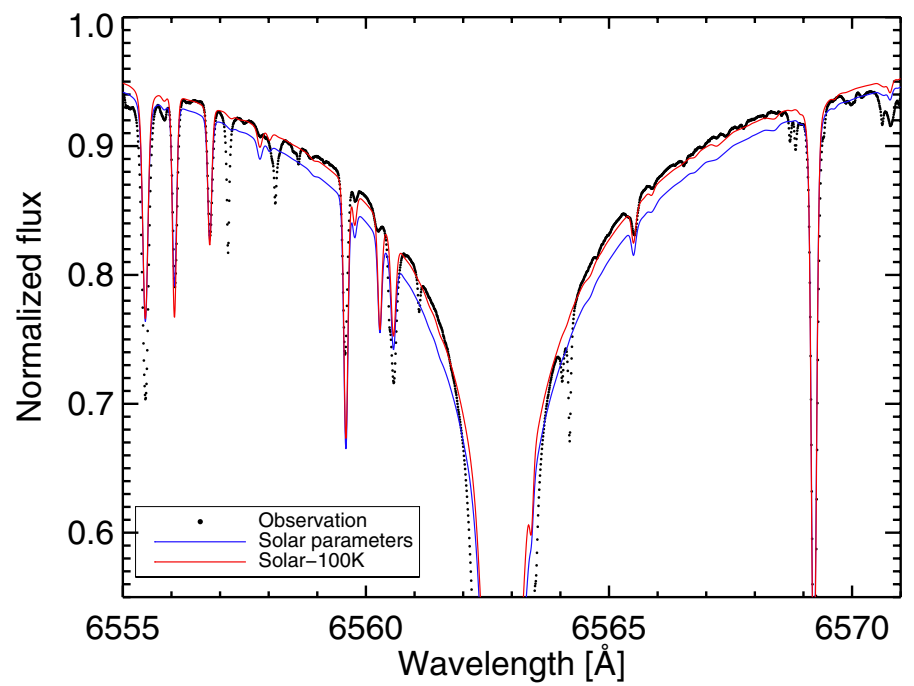

Fig. 1. Part of the observed solar spectrum (from the Kitt Peak Atlas, black dots) and the SME synthetic spectrum from a MARCS model with solar parameters (blue), as well as a MARCS model with $T_{\text {eff }}=5670 \mathrm{~K}$, else solar parameters (red). Obviously, the MARCS solar model produces a too wide $\mathrm{H} \alpha$ line.

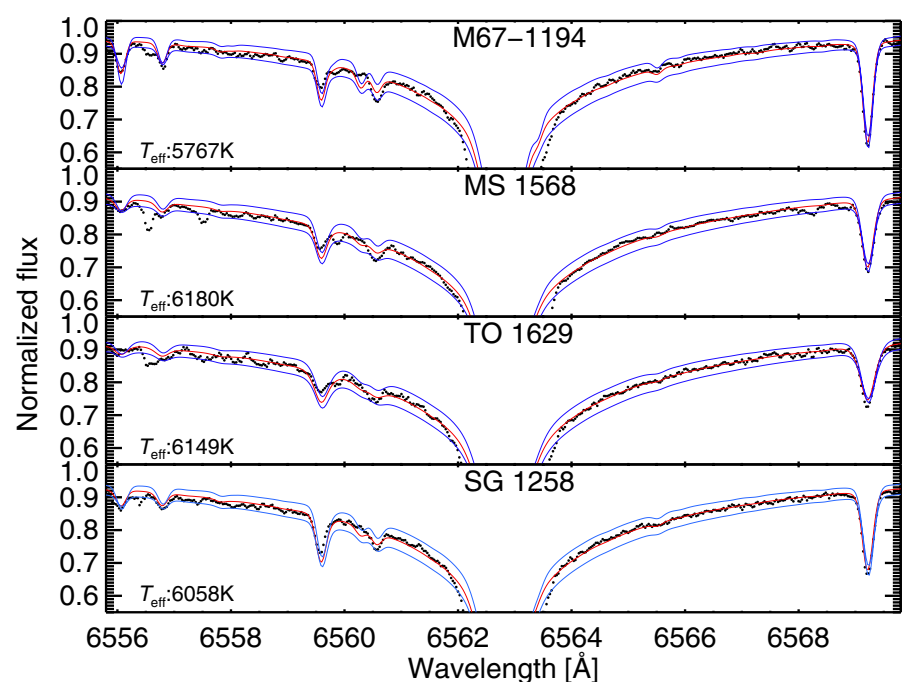

Fig. 2. Fits of the $\mathrm{H} \alpha$ wing profiles of four representative stars. The red lines show spectra of best fit models. Blue lines show the effects of temperature shifts by $\pm 100 \mathrm{~K}$. Effective temperatures given in the panels are corrected upwards by $100 \mathrm{~K}$ (see text).

Cayrel et al. (2011) who using ATLAS model atmospheres found an effective temperature from $\mathrm{H} \alpha 99 \mathrm{~K}$ too cool for the Sun and a systematic shift of this magnitude for solar-type stars with measured angular diameters. (We note, however, that Cornejo et al. 2012, using a similar method, find a solar temperature from $\mathrm{H} \alpha$ fits that is only $30 \mathrm{~K}$ too low. We also note that an independent attempt to fit the $\mathrm{H} \alpha$ profiles with the less detailed MAFAGSODF model atmospheres (Grupp 2004) and the outdated broadening data of Ali \& Griem (1965) leads to discrepancies with the observed solar wing profile that are much smaller.) Examples of our $\mathrm{H} \alpha$ fits are shown in Fig. 2. We have also made similar analyses of the $\mathrm{H} \beta$ line which supports a temperature correction of $100 \mathrm{~K}$. The $T_{\text {eff }}$ values resulting from fits of $\mathrm{H} \beta$ wings show an increased scatter when compared with results from colours and other temperature indicators (see below), which we ascribe to the less favourable placement of $\mathrm{H} \beta$ towards the edge of two

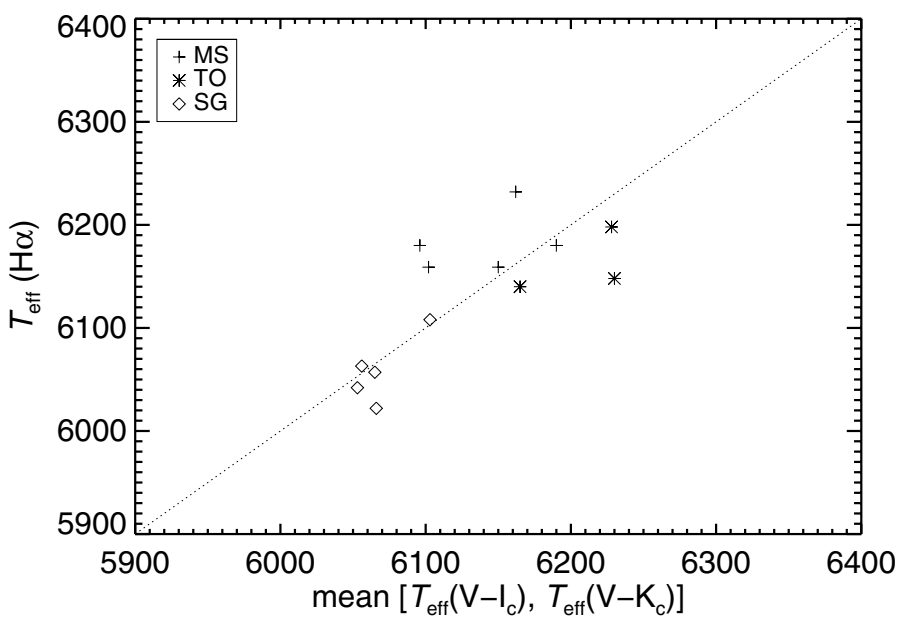

Fig. 3. Temperature estimates from the $\mathrm{H} \alpha$ profiles, corrected by $+100 \mathrm{~K}$, plotted vs. the mean of the temperatures derived from the $\left(V-I_{\mathrm{C}}\right)$ and $\left(V-K_{\mathrm{S}}\right)$ colours. Programme stars of different types are represented by different symbols. The dotted line is a $1-1$ line.

echelle orders and the higher density of blending metal lines in this part of the spectrum.

The resulting mean difference between the two photometric determinations $T_{\text {eff }}\left(V-K_{\mathrm{s}}\right)$ and $T_{\text {eff }}\left(V-I_{\mathrm{c}}\right)$ is $14 K \pm 48 K(\mathrm{~s} . \mathrm{d})$. In Fig. 3 we have plotted the temperatures determined from $\mathrm{H} \alpha$ vs. the means of the two photometric temperatures with the correction mentioned above. It is seen that these two temperature scales agree well. The mean difference is $-2 \mathrm{~K}$ and the standard deviation is $46 \mathrm{~K}$. There may be a tendency for the different stellar groups to behave somewhat differently, the MS stars being located higher in Fig. 3 than the SG and TO stars. Such systematic differences may reflect systematic errors in the relative temperatures and the effects of these on the abundances derived will be discussed below. We have adopted a mean of these two scales, the one based on colours and that based on $\mathrm{H} \alpha$ wings as temperature estimates for the programme stars.

In checking these temperatures, we have compared with ionisation temperatures, obtained from $\mathrm{Fe}$ I and Fe II lines, as well as Ti I and Ti II lines. The neutrals and the corresponding ions should give identical elemental abundances in the analyses. A good agreement is found, although with a zero-point shift of $61 \mathrm{~K} \pm 49 \mathrm{~K}$ (s.d.). One should note that these ionisation temperatures are essentially based on very few unsaturated lines from Fe II and Ti II, respectively. We have also compared with temperatures obtained by requiring identical abundances resulting from $\mathrm{Fe} I$ lines at different excitation. Here, we find a mean difference between the adopted temperatures and those obtained from the excitation equilibria in LTE of $25 \mathrm{~K} \pm 86 \mathrm{~K}$ (s.d.). We have finally also checked the temperature scale by using the method of line-depth ratios of Gray (1994) for lines from the same element with different excitation energies. Specifically, we selected the line pair Fe I $\lambda 5679$ and FeI $\lambda 6498$, and fitted the macro-turbulence parameter individually for each star, using a number of reasonably clean lines in the spectral interval to mimic the combination of effects of macroscopic motions in the atmospheres, rotation and instrumental profiles. We derived in this way effective temperatures that depart at a mean from the adopted ones by $-15 \mathrm{~K} \pm 50 \mathrm{~K}$ (s.d.).

In Fig. 4 we have plotted the mean of the spectroscopic temperature estimates, from ionisation equilibria ( $\mathrm{Fe}$ and $\mathrm{Ti}$ respectively), excitation equilibria (of Fe I), and relative line depths 


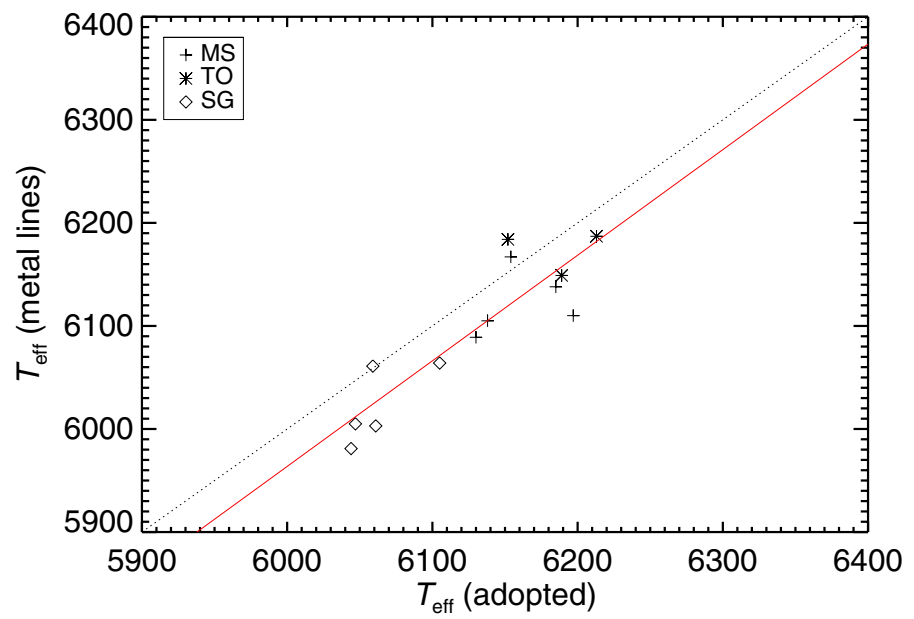

Fig. 4. Mean of the temperature estimates from ionisation equilibria (Fe and $\mathrm{Ti}$, respectively), excitation equilibria and relative line depths of metal lines, vs. the adopted effective temperatures. The 1-1 line (dots) and a regression line (red) are drawn. Symbols for different stellar groups are indicated.

(Fe I) relative to the adopted temperatures. The two scales are compatible at the $33 \pm 32 \mathrm{~K}$ level.

Altogether, we estimate that our adopted temperatures should be correct to within $\pm 30 \mathrm{~K}$ except for a possible systematic zero-point shift common to all stars and amounting to maximally $100 \mathrm{~K}$. In view of the differential character of our study, such a shift will not affect our final conclusions.

Surface gravities: the $\log g$ values were obtained from the $V$ magnitudes of the cluster stars (from Yadav et al. 2008), by applying corrections for interstellar extinction, adequate bolometric corrections, and estimating the stellar masses from the isochrones of VandenBerg et al. (2007). The errors in the differences $\log g-\log g$ (M 67-1194) are small and less than 0.03 dex and mainly due to errors in the photometry and possibly varying extinction, as well as errors in the mass estimates from the isochrones. A significant source of much greater errors would be the possible binarity of some of the stars, leading to underestimates of surface gravity which may amount to 0.3 dex. Such an effect would be of particular interest since it could be selective it might affect our stars classified as TO and SG stars more than the MS stars since the positions of the binaries are expected to be raised in the colour-magnitude diagram (CMD). In the CMD for the cluster of Sandquist (2004) we find most of the known binaries in such elevated positions, but none of our programme stars are among those, and they are also located low in the diagram. Thus, errors due to binarity are judged to only affect our results for few stars, and then with minor gravity (and abundance) effects. For the absolute values of $\log g$, additional errors enter due to errors in the value for M 67-1194, estimated by (Önehag et al. 2011 ) to be about 0.04 dex. These errors are, however, compensated for in the abundance analysis by the differential approach chosen.

The stars and their fundamental parameters are listed in Table 1.

\subsection{Abundances}

The abundances were derived from the line strengths of a sample of absorption lines, selected to be generally on the linear part of the curve of growth, $\log \left(W_{\text {eq }} / \lambda\right) \leq-5$, and to be relatively free of blends, as judged from the Kitt Peak Solar Atlas
Table 1. Fundamental parameters for the programme stars.

\begin{tabular}{lcccc}
\hline \hline Star & Group & $T_{\text {eff }}[\mathrm{K}]$ & $\log (g)\left[\mathrm{cm} \mathrm{s}^{2}\right]$ & $\xi_{\mathrm{t}}\left[\mathrm{km} \mathrm{s}^{-1}\right]$ \\
\hline 1194 & TWIN & 5780 & 4.44 & 1.0 \\
\hline 1116 & MS & 6131 & 4.27 & 1.4 \\
1221 & MS & 6155 & 4.30 & 1.4 \\
1265 & MS & 6138 & 4.25 & 1.4 \\
1367 & MS & 6197 & 4.22 & 1.4 \\
1568 & MS & 6185 & 4.24 & 1.4 \\
\hline 963 & TO & 6213 & 3.93 & 1.8 \\
1629 & TO & 6189 & 3.93 & 1.8 \\
1783 & TO & 6153 & 3.90 & 1.8 \\
\hline 863 & SG & 6048 & 3.83 & 1.7 \\
1188 & SG & 6060 & 3.85 & 1.8 \\
1248 & SG & 6044 & 3.81 & 1.6 \\
1258 & SG & 6061 & 3.82 & 1.6 \\
1320 & SG & 6106 & 3.82 & 1.8 \\
\hline
\end{tabular}

Notes. The type of the star is indicated. MS: Main-sequence stars, TO: Turn-off point stars, SG: Subgiant-branch stars.

and the Vienna Atomic Line Data Base (VALD, Kupka et al. 1999). Another important selection criterion was that lines used by Meléndez et al. (2009) in their study of solar twins were included if possible. For spectral lines where blends were suspected to occur in one of the wings, the abundance estimates were based on the other half of the line. In practice, synthetic spectra were fitted to the useful parts of the observed lines and then the equivalent widths were calculated from the synthetic spectra of corresponding single lines that were next compared with model single lines for varying abundances. In a few cases, fits with synthetic spectra were used directly, not only for deriving abundances from severely blended but important lines but also when using relatively strong lines affected by hyper-fine structure, and for the Li I $\lambda 6707$ doublet. All lines used in the abundance analysis are listed and commented on in Table A.1.

The two OI lines in the sample, the high-excitation temperature sensitive $\lambda 6158$ and the blended forbidden line $\lambda 6300$, are both rather weak in the temperature regime of the Sun and M 67-1194. Chi-square minimised synthetic fits were calculated for both lines using SIU (Reetz 1991), see also Önehag et al. (2011) for a more detailed description of the method and visualisation tool. Oscillator strengths for O I $\lambda 6300$ line and the $\mathrm{Ni}$ I blend were taken from Asplund et al. (2004) and Johansson et al. (2003), respectively. The solar O I and Ni I abundances used in the calculations were taken from Asplund et al. (2004).

The Li I doublet was found to vary considerably among the programme stars, and for four of them it was barely detectable at all. Some illustrative spectra are presented in Fig. 5.

Abundances were derived for each spectral line individually relative to those of M67-1194, with the exception of $\mathrm{Li}$ for which a direct calibration on the solar $\mathrm{Li}$ doublet was applied. We used MARCS model atmospheres (Gustafsson et al. 2008). The models were calculated for each star with appropriate $T_{\text {eff }}$ and $\log g$ and with solar abundances for all elements. The micro-turbulence parameter $\xi_{\mathrm{t}}$ was varied until the individual abundances for Fe I lines with different strengths were as equal as possible for each star (see Table 1). The resulting abundances are presented in Table A.2. Individual abundances for all lines are given in Table A.3.

The errors in the abundances derived due to errors in the fundamental parameters are presented in Table A.4. It is seen that the effects of errors in $T_{\text {eff }}$ are most significant, while effects 


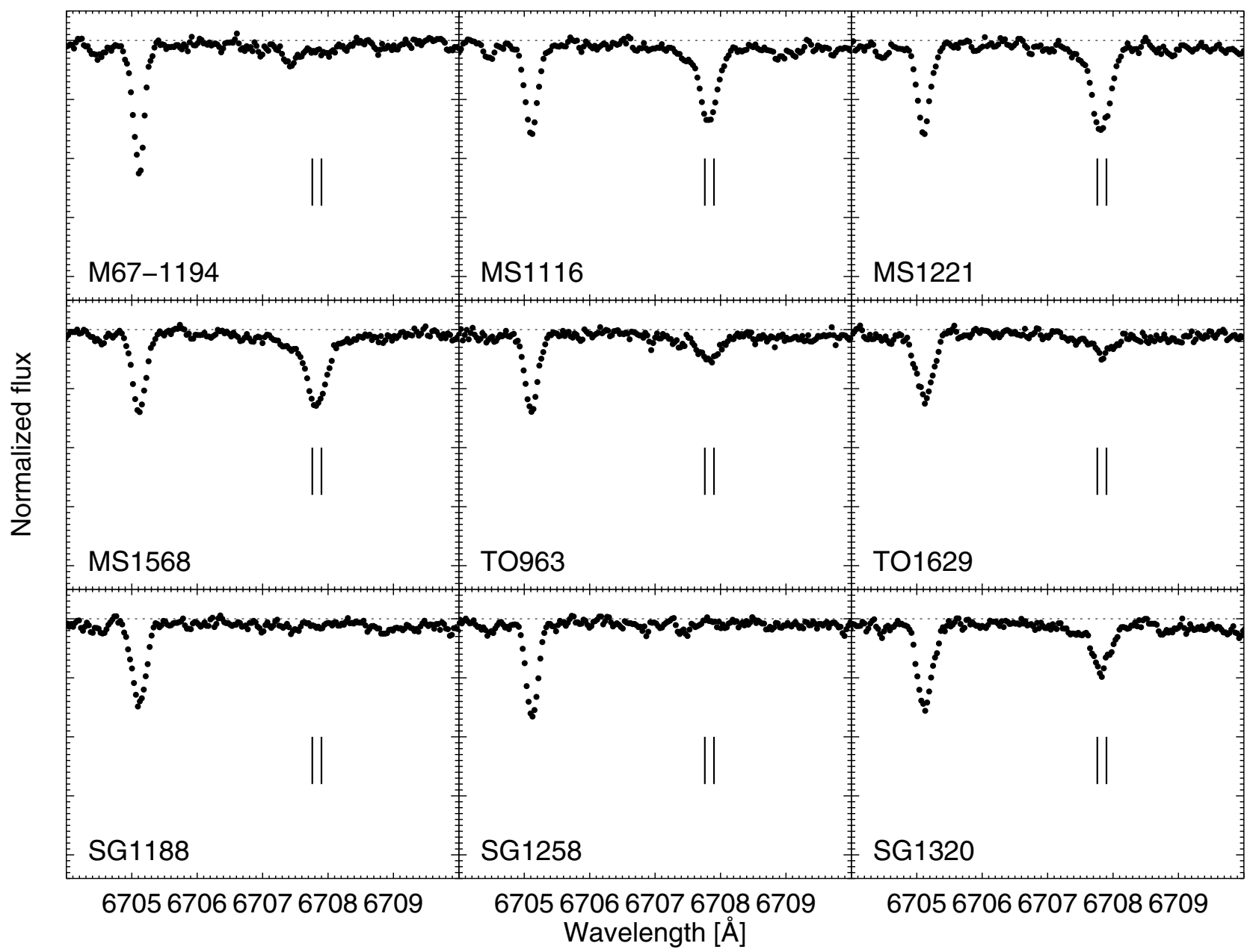

Fig. 5. Observed Li I $\lambda 6707$ doublets (marked by two vertical lines) in 8 stars (type and designation given) selected to demonstrate the full range of Li line strengths. The lines at $\lambda 6704.5$ and $\lambda 6705.1$ are Fe I lines.

due to errors in $\log g$ and in $\xi_{\mathrm{t}}$ are negligible for most elements. Errors in effective temperatures are minimised by the differential approach while a temperature scale error of e.g. $100 \mathrm{~K}$ would affect the absolute abundances considerably. The errors due to the inadequate assumptions of plane-parallel stratification, MLT convection, and LTE in the calculation of model atmospheres and spectra should also be minimised by the differential approach. However, they cannot be excluded from being of some significance, in particular for the differences in abundances obtained from the MS, TO, and SG stars, respectively (see below).

\section{Results and discussion}

In the following, two different but interlinked aspects of the results will be discussed: (1) The overall cluster abundances where means of the chemical abundance of each element for all programme stars are at focus; and (2) the systematic abundance differences between the three groups of stars, main-sequence stars (MS), turn-off point stars (TO), and subgiant-branch stars (SG). The first discussion is directly linked to the question of the abundance profile of the cluster, compared with that of the Sun and of Solar twins as explored by Meléndez et al. (2009) and Ramírez et al. (2009), while the second issue is related to studies of diffusion by Korn et al. (2007) and later authors referred to in Sect. 1. Finally, in the conclusions (Sect. 5) the interrelations between the conclusions drawn as regards the aspects (1) and (2) will be discussed.

\subsection{The abundance profile of the cluster and the dust-cleansing hypothesis}

As seen in Table A.2 (see also Panel 4 of Fig. 7) the abundances derived here for M67-1194 are fully consistent with those of Önehag et al. (2011) which reinforces the conclusion from that paper (albeit based on the same observational data) that the star M 67-1194 has an elemental composition very similar to that of the Sun. We may thus take this star as a solar proxy with solar-like effective temperature and gravity, and closer to the Sun in chemical composition than the Solar twins explored by Meléndez et al. (2009) and Ramírez et al. (2009).

In Fig. 6 the mean abundances for the cluster stars relative to those of M 67-1194 are plotted as a function of condensation temperature. Drawn in this figure is also the dashed red line showing the relation between abundance differences $(\Delta[\mathrm{X}(\mathrm{Fe}]$ (Sun - Solar twins)) of Meléndez et al. (2009). Clearly, the observed abundances agree with the hypothesis that the abundances of the cluster stars in general match those of M 67-1194 (and thus the Sun) better than those of the local twins. To put simple numbers on the certainty in this conclusion without referring to the somewhat uncertain estimated errors in the individual abundances, one may proceed as follows: we divide the full interval in condensation temperature $T_{\text {cond }}$ into three sub-intervals: $T_{\text {cond }}<1000 \mathrm{~K}, 1000 \mathrm{~K}<T_{\text {cond }}<1500 \mathrm{~K}$, and $1500 \mathrm{~K}<T_{\text {cond }}$. The ratios of the numbers of points above and below the red 


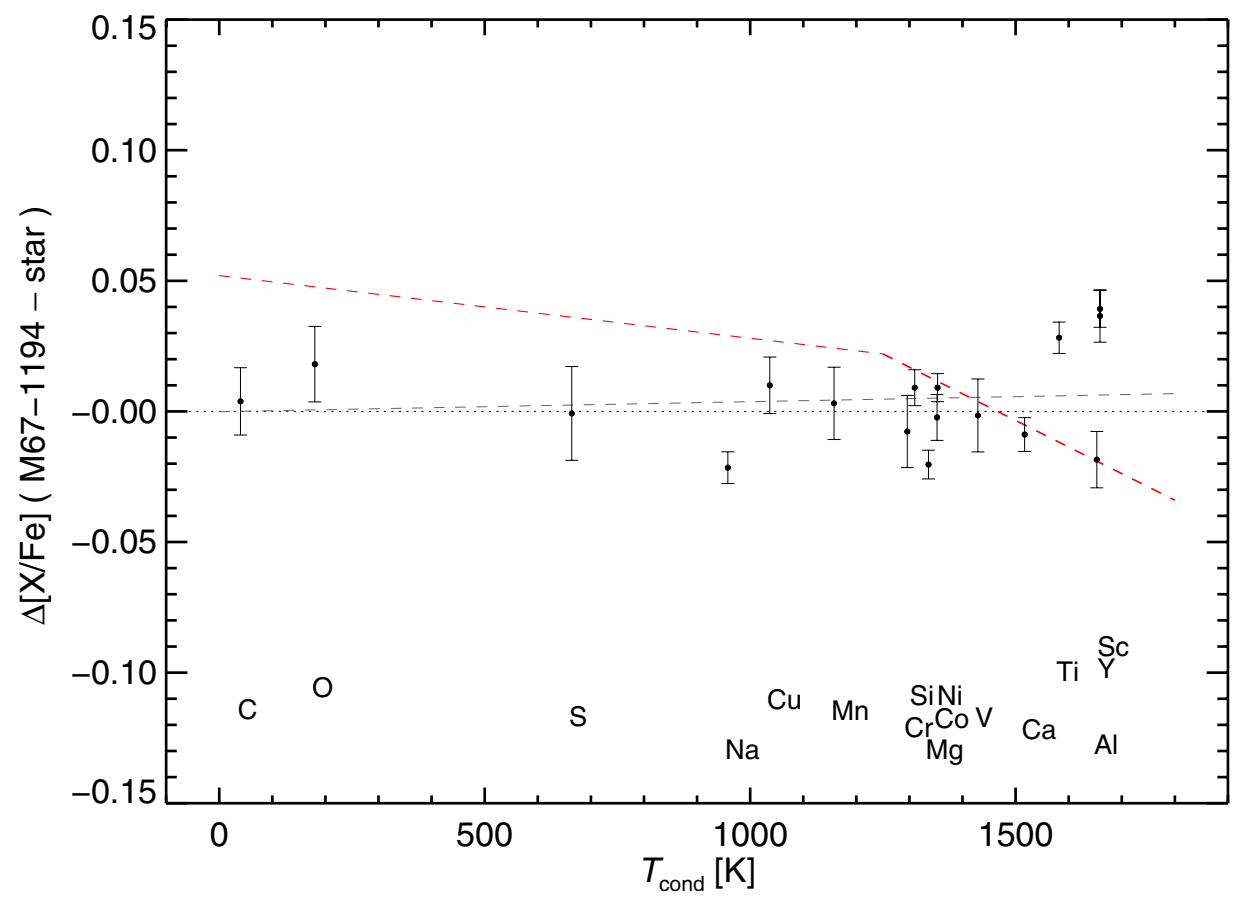

Fig. 6. Differences in logarithmic abundances between M67-1194 and the means for the other 13 programme stars of the present study, plotted vs. the condensation temperature of the elements (Lodders 2003). The red dashed line indicates the rough mean locus of the solar-solar twin abundances, according to Meléndez et al. (2009). The grey dashed line is a regression line to the data. Error bars represent the RMS errors in the means for the entire sample $(\sigma / \sqrt{N})$. dashed line in the diagram are, respectively $0 / 4,0 / 8$, and $4 / 1$. Similarly, for the point distribution around the line corresponding to the composition of M 67-1194 (the $x$-axis) the ratios are $2 / 2,4 / 4$, and $3 / 2$. Assuming that the points were randomly distributed around the respective lines and that the error distributions are symmetrical around the true values, one finds by simple combinatorics that the probability for the composition to be equal to that of M67-1194 (i.e. this analysis to show the observed configuration of points around the $x$-axis in the figure) is three orders of magnitude greater than the probability that it would be equal to the composition of the solar twins in the field.

A more detailed study requires errors to be estimated in the various relative abundance means $\langle[\mathrm{X} / \mathrm{Fe}]\rangle$ for the cluster stars. For the different elements we form these means for all our programme stars, and estimate the errors in the means as the standard deviation among the stars divided by $\sqrt{N}$, where $N$ is the number of stars. For elements where these errors are found to be less than 0.01 we adopt 0.01 , in order not to underestimate the errors. We next hypothetically assume the true mean abundances of M 67 to agree with the mean pattern of the Solar twins in the galactic field of Meléndez et al. (2009), i.e. to line up along the dashed line in Fig. 6 when compared with M 67-1194, our solar proxy, and that the apparent systematic difference between the observed values in the figure and the dashed relation is just circumstantial, due to the random errors in the analysis. When, using a Monte Carlo approach, we apply a gaussian scatter characterised by the errors in the means, we find that less than $0.1 \%$ of all sets of such perturbed abundances fall closer to the abscissa than to the dashed line, i.e. with an absolute slope of less than $1.6 \times 10^{-5} \mathrm{dex} / \mathrm{K}$ in the figure. Thus, the hypothesis that the set of true mean abundances is closer to that of the Solar twins than to that of M67-1194 is several orders of magnitude less probable than the opposite hypothesis. Indeed, even if the errors in $\langle[\mathrm{X} / \mathrm{Fe}]\rangle$ were assumed to be as high as 0.04 , a value which must be considered a severe overestimate, we find that the probability of the M 67 stars to show the pattern of M 67-1194 is 0.85, while the probability for them to show the pattern of the Solar twins is only 0.15 .
A natural question is now to what extent this result is sensitive to the possible systematic errors. The most important of these errors for our present results is possibilities of errors in the temperature scale, which affect the abundances derived for volatile elements (low condensation temperatures) more than the refractories, as a result of the high excitation energy of the lower levels of C I and S I lines used. An increase in the overall temperature scale by more than $200 \mathrm{~K}$ is needed to bring the M 67 stars anywhere near the abundance profile of the field dwarfs. In view of the discussion of the temperature scale in Sect. 3.1, we find such an error in the scale improbable. We thus conclude that the stars analysed in M 67 have a mean abundance profile resembling that of the very solar-like star M 67-1194, while they deviate systematically from the mean results obtained for the Solar twins in the field by Meléndez et al. (2009) and Ramírez et al. (2009).

It is not clear, however, that this mean profile is representative of all M 67 stars, as is displayed in Fig. 7 where abundance patterns are shown for the different groups of stars. The subgiants do not seem to unambiguously show the solar likeness that the other stars demonstrate. If the results for all stars are scrutinised individually, at least 3 stars may show deviating tendencies, with patterns somewhat more similar to those of the Solar twins in the field. These stars are M67-863, 1320, and 1248. It is noteworthy that these deviating stars are all classified as subgiants. It is not appropriate to conclude that these subgiants deviate because mixing of convective material from their interiors has erased departures at the surface, departures that could prevail for the MS and TO stars and could be due to diffusion in the stars or pollution of cleansed material from the proto-planetary disks. The convective zones of the subgiants at these relatively hot effective temperatures entail namely only about $2 \%$ of the stellar mass, a figure very similar to that of M 67-1194, which is why dilution by convective mixing of a possible abnormal surface composition should not affect the subgiants more than the solar-type dwarfs (we are indebted to Don VandenBerg for contributing these numbers). Instead, one may speculate that the deviating abundances may be related to the higher mass of the 

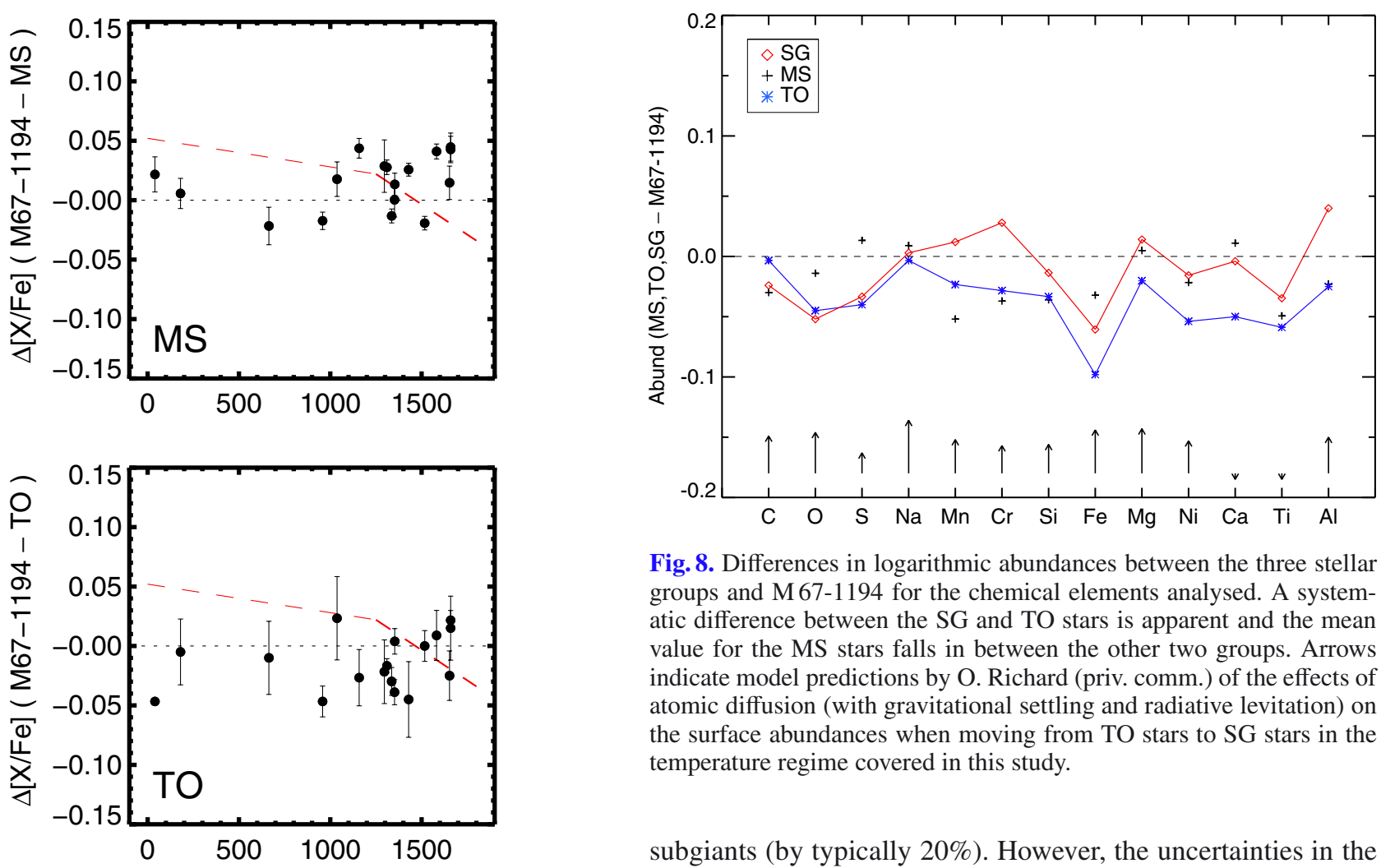

Fig. 8. Differences in logarithmic abundances between the three stellar groups and M 67-1194 for the chemical elements analysed. A systematic difference between the SG and TO stars is apparent and the mean value for the MS stars falls in between the other two groups. Arrows indicate model predictions by O. Richard (priv. comm.) of the effects of atomic diffusion (with gravitational settling and radiative levitation) on the surface abundances when moving from TO stars to SG stars in the temperature regime covered in this study.

subgiants (by typically 20\%). However, the uncertainties in the abundances, and the small sample of subgiants, do not admit us to draw any certain conclusions in those directions.

We note in passing that the SG star 1320 shows some anomalously small abundances from the OI $\lambda 6158$, SI $\lambda 6052$, and FeII $\lambda 5454$ lines, which might suggest a lower effective temperature than we have adopted. However, we do not find any evidence for a lower temperature from our different temperature estimates. It could also be suspected that the stellar spectrum is contaminated by a companion; however, we have not found any further evidence for this. If 1320 would be excluded from the sample, the subgiants would show a more solar-similar pattern in Fig. 7.

A possible explanation for our result in Fig. 6 is that the gas in dense proto-clusters might have been cleansed from dust by hot stars early on in the evolution of the cluster. Draine (2011) has explored the possibilities for dust to drift out of HII regions as a result of the stellar radiation pressure on the grains. For his static equilibrium models of HII regions he finds self-similar solutions, dependent on three parameters that characterise the stellar radiation, the gas to dust ratio and the product $Q_{0} \times n_{\mathrm{rms}}$, respectively. Here $Q_{0}$ is the number of photons that may ionise hydrogen emitted per second and $n_{\mathrm{rms}}$ is the rms density of protons within the ionised region. Adopting the estimate by Hurley et al. (2005) that M67 early on contained nearly 19000 solar masses in stars, one finds that the original Giant Molecular Cloud (GMC) from which the cluster formed had a mass around $10^{5} M_{\odot}$ if the time-integrated star formation efficiency $\epsilon$ was as high as $20 \%$, a number estimated for GMCs in the Milky Way (Murray 2011). With more traditional lower values of $\epsilon$ the mass would have been even greater. Adopting a standard stellar mass distribution function (Cox 2000), we then find that about 1 star formed in the cluster should have had a mass above 25 solar masses. For this case (corresponding to $Q_{0} \sim 10^{49}$ and $n_{\mathrm{rms}} \sim 500 \mathrm{~cm}^{-3}$ ) we find drift speeds of $0.1 \mathrm{~km} \mathrm{~s}^{-1}$ or more, and a characteristic time for the dust grains to drift out of the model HII region of 2 Myr, from Figs. 8 and 9 of Draine (2011). The latter value is fairly independent of the parameters of the

Fig. 7. Abundance differences (M67-1194 - group mean) where the means are taken for stars in the different groups, Main-sequence stars, Turn-off point stars and Subgiant stars, respectively. The solar-twin line of Meléndez et al. (2009) is indicated (red dashed). The panel in the bottom displays the differences Sun - M 67-1194. Error bars in the upper three panels represent the RMS errors in the group means $(\sigma / \sqrt{N})$. In the bottom panel the error bars represent line-to-line scatter. 


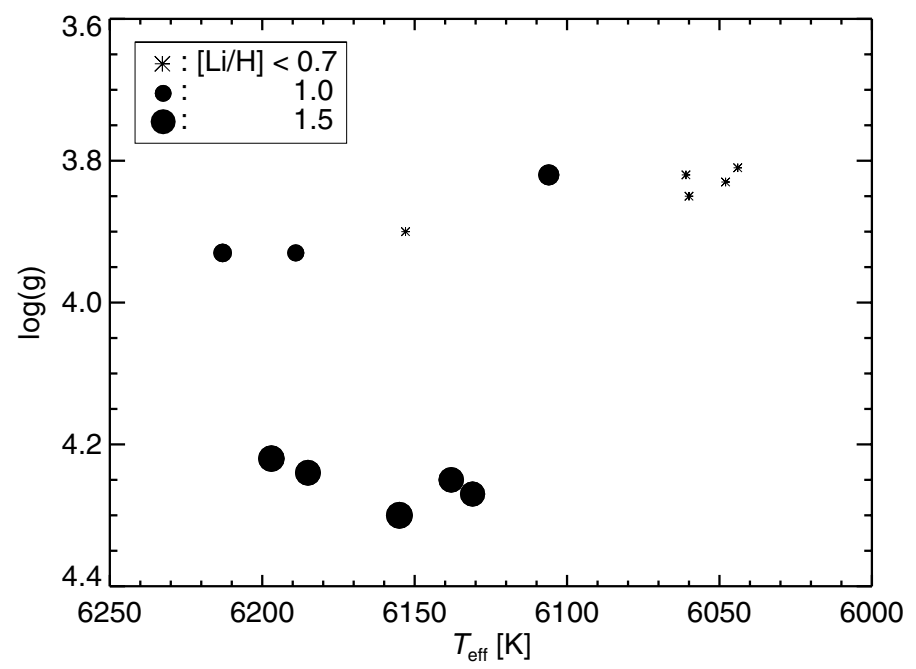

Fig. 9. Stars plotted in the $T_{\text {eff }}-\log g$ plane, with symbol sizes indicating the Li abundances. Asterisks show upper abundance limits.

model, although, as shown by Draine (2011) magnetic fields in the gas may increase the time by orders of magnitude. A higher total gas mass of the proto-cluster would lower the drift time in inverse proportion.

For the suggested dust cleansing of the proto-cluster cloud by hot stellar radiation to be the explanation for the composition found for the M67 stars, as compared with the solar twins in the galactic field which would then presumably have formed in less massive clusters where no high-mass star was formed, one must also assume a star-formation history in the proto-cluster cloud to occur in several generations, where the radiating massive star(s) formed before most of the lower-mass cluster stars. This could then be an example of a triggered star formation scenario, as discussed by Sharma et al. (2007), Jose et al. (2008), and Pandey et al. (2008, 2013), on the basis of studies of stellar distributions in OB associations. One could speculate that the sign of deviating abundances for the subgiants with their somewhat higher masses could be the result of an earlier star formation than for the solar-type dwarfs, at epochs when the cleansing had still not been efficient.

However, it is unclear whether this mechanism of dust cleansing at all gives the result observed. Indeed, as was pointed out by Draine (2011) the true HII regions are not static but their ionisation fronts are expanding with speeds that may well supersede the dust drift speeds. If the "collect and collapse process" of Elmegreen \& Lada (1977) were at play, where the neutral gas surrounding the HII region is collected between the ionisation front and its preceding shock front, and next collapses gravitationally, triggered by the pressure of the ionised gas (cf. simulations by Hosokawa \& Inutsuka 2005, 2006; and Dale et al. 2007), the dust drift may also not be swift enough to deplete the dust in the swept-up gas. It remains to explore whether the radiation-driven implosion (RDI) of molecular cloud condensations in the proto-cluster (Miao et al. 2006, and references therein) could admit dust depletion to take place.

The proposal that dust-cleansing is the explanation for the composition of $\mathrm{M} 67$ raises another question. We have found that the different stars in M 67 suggest a depletion of refractories by at the most about $20 \%$. Certainly, this does not correspond to a total depletion of all dust which once existed in the proto-cluster cloud, if the degrees of condensation typical of interstellar dust would apply. The latter depletions are often found to be one order of magnitude greater (Savage \& Sembach 1996). In this case, an efficient evaporation and mixing of the gas must have taken place before the programme stars were formed.

\subsection{The effects of diffusion in the stars and the lithium abundances}

In our analysis we attempted to trace another effect which is of interest to studies of stellar structure and evolution beyond classical quasi-hydrodynamic models. If the observed $[\mathrm{X} / \mathrm{H}]$ values, i.e. abundances relative to M 67-1194, of all elements except Li are averaged for all stars one finds the value $-0.017 \pm 0.003$ (the error being the mean error in the mean), while the corresponding predicted value from the model calculations of O. Richard (private communication) with atomic diffusion (but without additional mixing) is $-0.028 \pm 0.002$. Here, his models for an age of M 67 of $3.7 \mathrm{Gyr}$ were adopted. These values are calculated relative to our standard star M 67-1194 for which $[\mathrm{X} / \mathrm{H}]$ is set to zero; this star is cooler and has therefore a considerably deeper convection zone than most of our programme stars which is why the diffusion effects are expected to be smaller. The corresponding observed mean for the TO and MS stars, i.e. with the five SG stars with their deeper convection zones excluded, is, as one would expect if diffusion is active, absolutely larger, $-0.026 \pm 0.004$ which is compatible with the model predictions. A differential study of TO and MS stars relative to SG stars gives an observed difference in the means of $-0.021 \pm 0.007$ which again is compatible with the predictions. Thus, these different tests seem to suggest that diffusion is active. However, the diffusion models also predict selective effects when different elements are compared. Among the elements studied here, one would thus expect diminishing abundances of $\mathrm{Ca}$ and $\mathrm{Ti}$ by about 0.01 dex when the stars proceed from the TO to the $S G$, a behaviour in contrast to the increase in atmospheric abundances shown by the other elements. This effect is not demonstrated by our data which instead indicate a similar behaviour for $\mathrm{Ca}$ and $\mathrm{Ti}$ as for, e.g., Fe.

These effects are illustrated for the individual elements in Fig. 8 where also the effects of atomic diffusion according to the model calculations by Richard are indicated by arrows. Corrections of the temperatures for the groups relative to one another might possibly explain the abundance differences seen in the figure: a possible increase in $T_{\text {eff }}$ by $50 \mathrm{~K}$ for the TO stars relative to the $\mathrm{SG}$ stars, as may be suggested by the ionisation equilibria of Fe and Ti (cf. Fig. 4), would explain the effects for most of the temperature-sensitive species (Mg I, Ca I, Ti I, Fe I etc.).

We conclude that our results suggest the presence of diffusion-induced heavy-element abundance differences in solarmetallicity stars. The correspondence between theory and observation is acceptable, albeit not equally so for all elements. Some precaution must be exercised, since systematic errors in even differential chemical analyses of stars are still noticeable. As one example, brought forward by the referee, one may take the case of the binary $16 \mathrm{Cyg} \mathrm{A}$ and $\mathrm{B}$, two solar analogues which have recently been analysed on the basis of spectra of very high quality, independently by Ramírez et al. (2011) and Schuler et al. (2011). While the former found an overabundance of all heavy elements of the A component by $0.04 \mathrm{dex}$ as compared to the B component, the latter group obtained more similar or identical abundances for the two components. This illustrates the fact that even accurate analyses, based on first-class data, may suffer from systematic errors that are significant when accuracies better than 0.05 dex are needed. Therefore, the differences traced by us for the subgroups of the M67 stars must still be regarded as 
somewhat preliminary. Independent verification with still better data is important.

We note that according to the model predictions, the metallicities of all observed stars have to be corrected upwards if one is interested in the original composition of M 67. Judging from the Richard models, its likely value lies close to +0.06 (cf. the Sun's original composition of +0.05 , Lodders 2010). Such an increase in the interior metallicity of stars in M 67 also improves a fit of the CMD at the TO region by producing a gap representing the occurrence of sustained core convection on the main sequence (see the discussion of VandenBerg et al. 2007).

Lithium may be used as a sensitive tracer of the mixing history in the outer layers of solar-type stars. In Fig. 9 we have plotted our Li abundance estimates as different symbol sizes in a $T_{\text {eff }}-\log g$ diagram. Our results are fairly consistent with those of Pasquini et al. (2008) who measured Li abundances for a great number of M 67 stars, although their results, based on more spectra of lower quality, show a greater scatter in abundances for stars in the parameter space of our MS stars. The errors in our abundances are typically about $0.1 \mathrm{dex}$, or greater for the stars with lowest abundances due to the weakness of the Li I $\lambda 6707$ doublet (here our values only represent upper limits, as indicated in the figure). It is seen that the abundances for the MS stars are typically 1.5 dex higher, with only a small scatter, than the solar photospheric abundance, which may be compared with the difference between the solar system logarithmic meteoritic value and the photospheric value of 2.2, as listed by Asplund et al. (2009). Note that our $[\mathrm{Li} / \mathrm{H}]$ values given in tables, figures and in the text above are all logarithmic values, determined directly relative to the Sun, i.e. unlike for the other abundances M 67-1194 has not been used for Li as a solar proxy. A correlation between stellar mass and Li abundance has been found in studies of M 67 (Pace et al. 2012; Li et al. 2012). The higher Li abundance determined for our MS stars are, according to these studies, likely reflecting the higher mass for the MS stars compared to the Sun. For the TO stars there is a significant spread towards lower values. Among the five SG stars, we find a very large scatter, 1.2 dex, in lithium abundances. Star 1320, discussed in Sect. 4.1, seems unmixed while the four other SG stars retain little if any of their original lithium. This mixing would be non-standard in nature and may be related to the physical processes giving rise to the lithium dip seen in younger open clusters. Lithium itself may obviously serve to constrain the mixing scenario.

\section{Conclusions}

Our main result is the following: a dominant fraction of the stars in M 67 show abundance patterns more similar to the Sun than most solar twins in the Solar neighbourhood do. This supports the conclusion that the Sun was formed in a dense stellar environment. A probable but not certain reason for this specific pattern is the action of dust cleansing in the primordial states of the cluster, as well as in the proto-stellar cloud that formed the Sun.

Even if there are several independent indications that the Sun was once formed in a dense environment it remains to be explained why its degree of depletion of refractories agrees quantitatively with that of the M 67 stars. A tempting, though not unproblematic, solution of this problem would be that the Sun originated in M67. In order for this to be reasonable, one must explain why the Sun now has an orbit close to the Galactic plane, while M 67 is presently high above the plane at a $z$ distance of $450 \mathrm{pc}$. As shown by Pichardo et al. (2012), the Sun could hardly have elapsed from the cluster into its present orbit without loosing significant parts of its planetary system. It remains to be seen if the converse scenario, where the cluster was formed in an orbit close to the plane and lost the Sun before it was diverted into a higher-altitude orbit, e.g. as a result of a collision with a Giant Molecular Cloud or through the effect of Galactic spiral arms is viable.

Recently Meléndez et al. (2012) studied the solar twin HD 101364 (HIP 56948) and found it to have a composition very close to solar, thus departing significantly from the composition of most twins in the solar neighbourhood. The authors estimated the age of HD 101364 to $3.52 \pm 0.68 \mathrm{Gyr}$, which is compatible with the age estimates for M67. There might be a possibility that this star once formed in the cluster - the typical distance in between the almost 20000 stars that have left it (Hurley et al. 2005 ) since it formed can be estimated to be about $100 \mathrm{pc}$, provided that they are distributed like Pop I stars in the Galactic disk at a distance $7 \mathrm{kpc}-9 \mathrm{kpc}$ from the Galactic centre. This would make the chances of finding a star brighter than 9th magnitude $(V($ HD 101364$)=8.67)$ non-negligible. We note, however, that the velocity perpendicularly to the Galactic plane of this star is only $W=-5.93 \mathrm{~km} \mathrm{~s}^{-1}$ which does not admit it to reach the height above the plane that M67 has today, while its $U$ velocity is high, $36 \mathrm{~km} \mathrm{~s}^{-1}$. Anyhow, this interesting star is valuable as a template for comparative studies of solar twins in M 67 and other clusters.

Several alternative explanations for the systematic abundance difference between the Sun and the field twins were discussed by Meléndez et al. (2009) and Gustafsson et al. (2010). An interesting possibility is that some stars, like the Sun, only had a shallow convective zone when the gas of the protoplanetary disk depleted by planetary formation was dumped onto its surface, and never were fully mixed after that. Such an unconventional solar formation history could result from rapid episodic accretion onto a small stellar core, as has been suggested by Baraffe \& Chabrier (2010). Our M 67 project was partly devised as a test of this possibility - an outcome proving that stars in a dense environment all showed the solar abundance profile would then suggest the dust-cleansing hypothesis to be the right one and disprove the local gas-disk pollution hypothesis. True enough, the result of the present study supports the first hypothesis. However, it is still possible that the local-disk pollution hypothesis is valid. One might for instance argue that the proto-stellar disks of stars in dense environments are affected by radiation, or even gravitational disturbances, from nearby stars, such that the instabilities in the disk are accentuated, promoting rapid episodic accretion onto the star.

Our tentatitve detection of systematic heavy-element abundance differences among stars in a solar-metallicity cluster opens up the possibility of observationally constraining (and subsequently correcting for) the amount of atomic diffusion affecting the surface abundances of late-type stars like the Sun. It remains to combine this finding with the Li depletion data into a coherent picture.

In the discussion above we have tried to separate the two phenomena observed: (1) the similarity between the overall abundance profile of M 67 and that of the Sun, being different from the abundance profile of the twins in the solar neighbourhood; and (2) the indications that the atmospheres of TO and MS stars seem to be depleted in heavy elements as compared to those of the SG stars. However, we have also pointed out the fact that these two phenomena are interrelated, since the composition of the SG stars according to diffusion models are presumably less affected by diffusion and thus should show a composition closer to the cloud from which the cluster once formed. As we saw in 
Fig. 7 there is a tendency, yet with the present data of marginal significance, for the SG stars not to clearly show the composition profile of the Sun. Instead they could be more similar to the twins in the solar neighbourhood. Now, if the SG stars are considered to be essentially free of the diffusion effects, could one not argue that what we see in the TO and MS stars is only reflecting one phenomenon: the effects of diffusion (or other element separation) intrinsic to the stars, and that the original abundance profile indeed was quite similar to that of the field twins? The main problem with that argument is then why the diffusion effects found in M 67 are not shown by the field solar twins except for the Sun itself. One could speculate that, e.g., the rotation of the cluster stars, or their primordial magnetic fields, or their accretion history, were systematically different from that of the field stars (but not of the Sun), leading to different and more efficient element separation in these stars than for the field stars. Such hypotheses have, however, little support theoretically or observationally, and could as well go the other way. If alternatively the dust cleansing scenario is advocated to explain the abundance profile of the TO and MS stars, one must explain why it does not seem to have affected the SG stars as much. The hypothesis that the SG stars, due to their systematically higher masses, were formed before the cloud was fully cleansed or that their protoplanetary disks evolved differently as compared to the lowermass TO and MS stars, are also rather ad hoc. In conclusion it seems clear that a study at higher resolution and $\mathrm{S} / \mathrm{N}$, and with more cluster stars, is needed to settle these issues.

Our two findings, indicating effects of fractionation of chemical elements in star-forming regions or even within the individual solar-mass stars, have important consequences for studies where solar-type stars are used to explore the chemical evolution of the Galaxy. Obviously, it cannot be taken for granted that solar-type stars, in spite of their comparatively well-mixed surface layers, fully reflect the chemical composition of their primordial gas and dust clouds.

Acknowledgements. Several colleagues have contributed importantly to the present paper. Eric Stempels devised the method to correct the observed spectra for light-contamination in the fibre bundles of FLAMES-UVES. Frank Grundahl supplied photometric data and fundamental-parameter estimates for the programme stars. Olivier Richard allowed us to use results from calculations of atomic-diffusion effects in models of stellar evolution representing M67. Kjell Eriksson calculated effects of $\log g$ and microturbulence variations on the $V-K_{\mathrm{s}}$ and $V-I_{\mathrm{c}}$ colours and Don VandenBerg provided data on evolutionary models of solar-mass stars. We also thank the anonymous referee for valuable comments on the manuscript.

\section{References}

Ali, A. W., \& Griem, H. R. 1965, Phys. Rev., 140, A1044

Asplund, M., Grevesse, N., Sauval, A. J., Allende Prieto, C., \& Kiselman, D. 2004, A\&A, 417, 751

Asplund, M., Grevesse, N., Sauval, A. J., \& Scott, P. 2009, ARA\&A, 47, 481

Baraffe, I., \& Chabrier, G. 2010, A\&A, 521, A44

Barklem, P. S., Piskunov, N., \& O’Mara, B. J. 2000, A\&A, 363, 1091

Biazzo, K., Pasquini, L., Bonifacio, P., Randich, S., \& Bedin, L. R. 2009, Mem. Soc. Astron. It., 80, 125

Casagrande, L., Ramírez, I., Meléndez, J., Bessell, M., \& Asplund, M. 2010, A\&A, 512, A54

Cayrel, R., van't Veer-Menneret, C., Allard, N. F., \& Stehlé, C. 2011, A\&A, 531, A83
Cornejo, D., Ramirez, I., \& Barklem, P. S. 2012 [arXiv: 1206.0750] Cox, A. N. 2000, Allen's astrophysical quantities (New York: AIP Press) Cutri, R. M., Skrutskie, M. F., van Dyk, S., et al. 2003, VizieR Online Data Catalog: II $/ 246$

Dale, J. E., Clark, P. C., \& Bonnell, I. A. 2007, MNRAS, 377, 535

Draine, B. T. 2011, ApJ, 732, 100

Elmegreen, B. G., \& Lada, C. J. 1977, ApJ, 214, 725

Gray, D. F. 1994, PASP, 106, 1248

Grupp, F. 2004, A\&A, 426, 309

Gruyters, P., Korn, A. J., Richard, O., et al. 2013, A\&A, 555, A31

Gustafsson, B., Edvardsson, B., Eriksson, K., et al. 2008, A\&A, 486, 951

Gustafsson, B., Meléndez, J., Asplund, M., \& Yong, D. 2010, Ap\&SS, 328, 185

Hobbs, L. M., \& Thorburn, J. A. 1991, AJ, 102, 1070

Hosokawa, T., \& Inutsuka, S.-I. 2005, ApJ, 623, 917

Hosokawa, T., \& Inutsuka, S.-I. 2006, ApJ, 646, 240

Hurley, J. R., Pols, O. R., Aarseth, S. J., \& Tout, C. A. 2005, MNRAS, 363, 293

Johansson, S., Litzén, U., Lundberg, H., \& Zhang, Z. 2003, ApJ, 584, L107

Jose, J., Pandey, A. K., Ojha, D. K., et al. 2008, MNRAS, 384, 1675

Korn, A. J., Grundahl, F., Richard, O., et al. 2007, ApJ, 671, 402

Kupka, F., Piskunov, N., Ryabchikova, T. A., Stempels, H. C., \& Weiss, W. W. 1999, A\&AS, 138, 119

Kurucz, R. L., Furenlid, I., Brault, J., \& Testerman, L. 1984, Solar flux atlas from 296 to $1300 \mathrm{~nm}$

Li, T. D., Bi, S. L., Chen, Y. Q., et al. 2012, ApJ, 746, 143

Lind, K., Korn, A. J., Barklem, P. S., \& Grundahl, F. 2008, A\&A, 490, 777

Lodders, K. 2003, ApJ, 591, 1220

Lodders, K. 2010, in Principles and Perspectives in Cosmochemistry, eds. A. Goswami, \& B. E. Reddy, 379

Majaess, D. J., Turner, D. G., Lane, D. J., \& Krajci, T. 2011, J. Am. Assoc. Variable Star Observers (JAAVSO), 39, 219

Meléndez, J., Asplund, M., Gustafsson, B., \& Yong, D. 2009, ApJ, 704, L66

Meléndez, J., Bergemann, M., Cohen, J. G., et al. 2012, A\&A, 543, A29

Miao, J., White, G. J., Nelson, R., Thompson, M., \& Morgan, L. 2006, MNRAS, 369,143

Mucciarelli, A., Salaris, M., Lovisi, L., et al. 2011, MNRAS, 412, 81

Murray, N. 2011, ApJ, 729, 133

Nordlander, T., Korn, A. J., Richard, O., \& Lind, K. 2012, ApJ, 753, 48

Önehag, A., Korn, A., Gustafsson, B., Stempels, E., \& Vandenberg, D. A. 2011, A\&A, 528, A85

Pace, G., Pasquini, L., \& François, P. 2008, A\&A, 489, 403

Pace, G., Castro, M., Meléndez, J., Théado, S., \& do Nascimento, Jr., J.-D. 2012, A\&A, 541, A150

Pandey, A. K., Sharma, S., Ogura, K., et al. 2008, MNRAS, 383, 1241

Pandey, A. K., Samal, M. R., Chauhan, N., et al. 2013, New Astron., 19, 1

Pasquini, L., Biazzo, K., Bonifacio, P., Randich, S., \& Bedin, L. R. 2008, A\&A, 489, 677

Pichardo, B., Moreno, E., Allen, C., et al. 2012, AJ, 143, 73

Piskunov, N. E., \& Valenti, J. A. 2002, A\&A, 385, 1095

Ramírez, I., Meléndez, J., \& Asplund, M. 2009, A\&A, 508, L17

Ramírez, I., Meléndez, J., Cornejo, D., Roederer, I. U., \& Fish, J. R. 2011, ApJ, 740,76

Randich, S., Sestito, P., Primas, F., Pallavicini, R., \& Pasquini, L. 2006, A\&A, 450,557

Reetz, J. K. 1991, Diploma Thesis, Universität München

Sandquist, E. L. 2004, MNRAS, 347, 101

Sarajedini, A., Dotter, A., \& Kirkpatrick, A. 2009, ApJ, 698, 1872

Savage, B. D., \& Sembach, K. R. 1996, ApJ, 470, 893

Schuler, S. C., Cunha, K., Smith, V. V., et al. 2011, ApJ, 737, L32

Sharma, S., Pandey, A. K., Ojha, D. K., et al. 2007, MNRAS, 380, 1141

Tautvaišiene, G., Edvardsson, B., Tuominen, I., \& Ilyin, I. 2000, A\&A, 360, 499

Taylor, B. J. 2007, AJ, 133, 370

Valenti, J. A., \& Fischer, D. A. 2005, ApJS, 159, 141

Valenti, J. A., \& Piskunov, N. 1996, A\&AS, 118, 595

VandenBerg, D. A., Gustafsson, B., Edvardsson, B., Eriksson, K., \& Ferguson, J. 2007, ApJ, 666, L105

Yadav, R. K. S., Bedin, L. R., Piotto, G., et al. 2008, A\&A, 484, 609

Yakut, K., Zima, W., Kalomeni, B., et al. 2009, A\&A, 503, 165

Yong, D., Carney, B. W., \& Teixera de Almeida, M. L. 2005, AJ, 130, 597 
A. Önehag et al.: Abundances and diffusion in M 67 stars

Appendix A: Tables of lines, derived abundances, and error estimates

Table A.1. Lines used in the abundance analysis.

\begin{tabular}{|c|c|c|c|c|c|}
\hline Elem. & $\lambda[\AA]$ & $\chi[\mathrm{eV}]$ & $\log g f$ & $W_{\mathrm{eq} \odot}[\mathrm{m} \AA]$ & \\
\hline Li I & 6707.761 & 0.00 & 0.167 & 4.1 & synth \\
\hline CI & 5380.337 & 7.68 & -1.842 & 23.8 & $b$ \\
\hline O I & 6158.186 & 10.74 & -0.409 & 4.8 & siu \\
\hline O I & 6300.304 & 0.00 & -9.819 & 6.2 & siu \\
\hline $\mathrm{NaI}$ & 6154.226 & 2.10 & -1.560 & 40.6 & $b$ \\
\hline $\mathrm{NaI}$ & 6160.747 & 2.10 & -1.260 & 62.2 & $b$ \\
\hline $\mathrm{Mg} \mathrm{I}$ & 5711.088 & 4.35 & -1.833 & 108.0 & $s$ \\
\hline $\mathrm{Mg} \mathrm{I}$ & 5183.604 & 2.72 & -0.180 & & $s$, synth \\
\hline AlI & 6696.185 & 4.02 & -1.576 & 39.4 & $b$ \\
\hline $\mathrm{Al} \mathrm{I}$ & 6698.673 & 3.14 & -1.647 & 22.4 & $b$ \\
\hline $\mathrm{Si} \mathrm{I}$ & 5665.555 & 4.92 & -2.040 & 42.9 & \\
\hline Si I & 5690.425 & 4.93 & -1.870 & 53.3 & \\
\hline $\mathrm{Si}$ I & 6125.021 & 5.61 & -1.464 & 35.2 & \\
\hline Si I & 6131.852 & 5.62 & -1.615 & 26.0 & $b$ \\
\hline Si I & 6721.848 & 5.86 & -1.516 & 49.7 & \\
\hline S I & 6046.027 & 7.87 & -1.030 & 11.6 & $b$ \\
\hline S I & 6052.674 & 7.87 & -0.740 & 13.4 & \\
\hline S I & 6757.171 & 7.87 & -0.310 & 15.6 & $b$ \\
\hline $\mathrm{Ca} \mathrm{I}$ & 5590.114 & 2.52 & -0.571 & 96.2 & $b, s$ \\
\hline $\mathrm{Ca} \mathrm{I}$ & 5867.562 & 2.93 & -1.570 & 27.9 & \\
\hline $\mathrm{Ca} \mathrm{I}$ & 6166.439 & 2.52 & -1.142 & 73.8 & $s$ \\
\hline $\mathrm{Ca} \mathrm{I}$ & 6169.042 & 2.52 & -0.797 & 100.2 & $b, s$ \\
\hline Sc II & 5684.202 & 1.51 & -1.074 & 39.4 & $b$ \\
\hline Sc II & 6245.637 & 1.51 & -1.030 & 36.6 & $b$ \\
\hline Ti I & 5219.702 & 0.02 & -2.292 & 30.2 & $b$ \\
\hline Ti I & 6126.216 & 1.07 & -1.425 & 24.1 & \\
\hline Ti I & 6258.102 & 1.44 & -0.355 & 52.9 & \\
\hline Ti II & 5490.690 & 1.57 & -2.430 & 23.6 & \\
\hline Ti II & 6491.561 & 2.06 & -1.793 & 41.4 & $b$ \\
\hline V I & 5670.853 & 1.08 & -0.420 & 20.3 & \\
\hline V I & 6090.214 & 1.08 & -0.062 & 35.7 & \\
\hline $\mathrm{CrI}$ & 5238.961 & 2.71 & -1.305 & 18.2 & $b$ \\
\hline $\mathrm{CrI}$ & 5287.178 & 3.44 & -0.907 & 12.4 & $b$ \\
\hline Mn I & 5377.637 & 3.84 & -0.109 & 50.3 & \\
\hline $\mathrm{FeI}$ & 5295.312 & 4.41 & -0.967 & 91.4 & $b$ \\
\hline $\mathrm{Fe} \mathrm{I}$ & 5522.446 & 4.21 & -1.514 & 65.2 & \\
\hline $\mathrm{Fe} \mathrm{I}$ & 6151.617 & 2.18 & -1.530 & 125.4 & \\
\hline $\mathrm{Fe} \mathrm{I}$ & 6229.226 & 2.85 & -2.482 & 84.7 & \\
\hline $\mathrm{FeI}$ & 6498.938 & 0.96 & -2.027 & 108.6 & $b$ \\
\hline $\mathrm{Fe}$ II & 5414.073 & 3.22 & -3.645 & 32.0 & $b$ \\
\hline Co I & 5342.695 & 4.02 & 0.690 & 35.4 & $b$ \\
\hline Co I & 5530.774 & 1.71 & -2.060 & 17.2 & $b$ \\
\hline Co I & 5647.234 & 2.28 & -1.560 & 14.8 & \\
\hline $\mathrm{Ni} I$ & 5589.357 & 3.90 & -1.140 & 31.2 & $b$ \\
\hline Ni I & 6086.276 & 4.27 & -0.530 & 46.2 & $b$ \\
\hline $\mathrm{Ni} I$ & 6130.130 & 4.27 & -0.960 & 23.4 & \\
\hline $\mathrm{Ni} I$ & 6204.600 & 4.09 & -1.100 & 23.2 & \\
\hline $\mathrm{Ni} I$ & 6223.981 & 4.11 & -0.910 & 29.6 & \\
\hline Ni I & 6772.313 & 3.66 & -0.980 & 52.7 & \\
\hline $\mathrm{Cu} \mathrm{I}$ & 5218.197 & 3.82 & 0.476 & 58.0 & $b, s$ \\
\hline $\mathrm{Cu} \mathrm{I}$ & 5220.066 & 3.82 & -0.448 & 15.2 & $b$ \\
\hline Y II & 4900.120 & 1.03 & -0.090 & 59.2 & $b, s$ \\
\hline Y II & 5087.416 & 1.08 & -0.170 & 50.6 & $b, s$ \\
\hline
\end{tabular}

Notes. Column description (left to right): Atomic element, wavelength, excitation energy, oscillator strength according to VALD, measured equivalent width from the Kitt Peak Solar Atlas, comments. $b$ : blend in wing(s), parts of line used to calculate $\mathrm{W}_{\text {eq }}$; $s$ : strong- or intermediate strong line in stellar spectra, $\log \left(W_{\mathrm{eq}} / \lambda\right)>-5$; synth: abundance determined directly from synthetic fit; siu: abundance determination and synthetic fit made in SIU. 


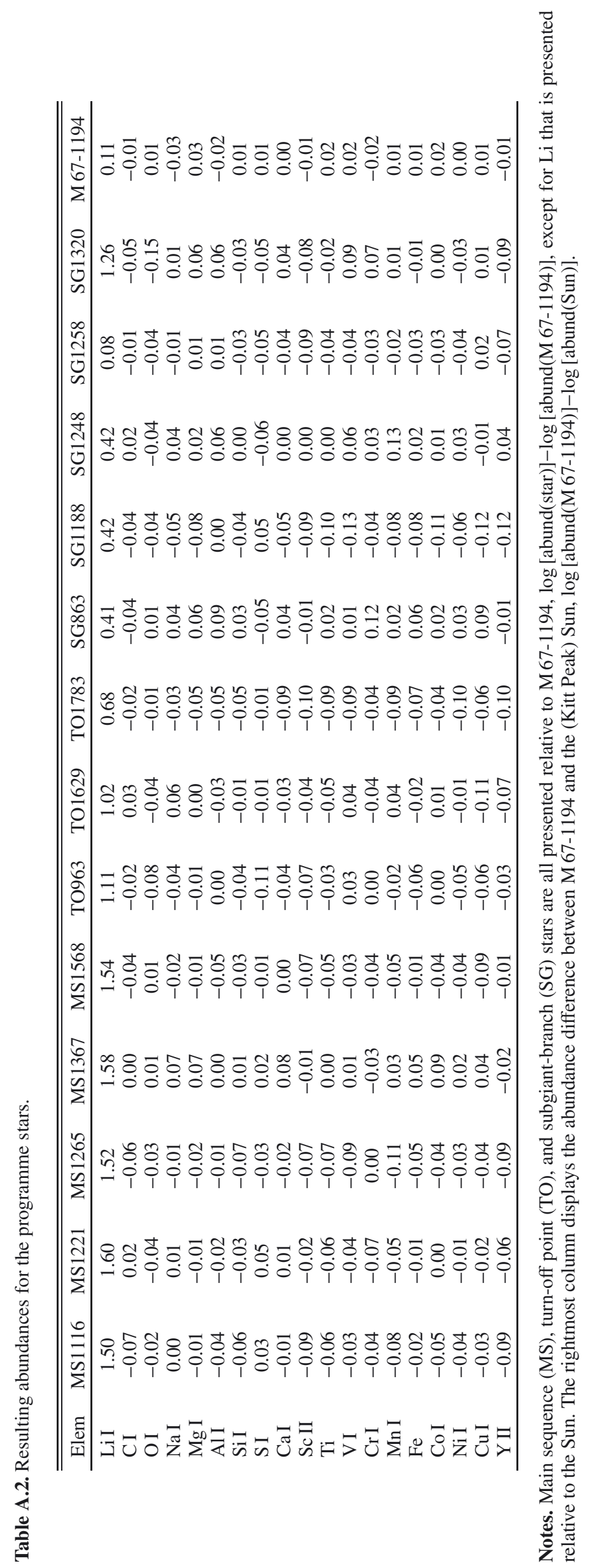




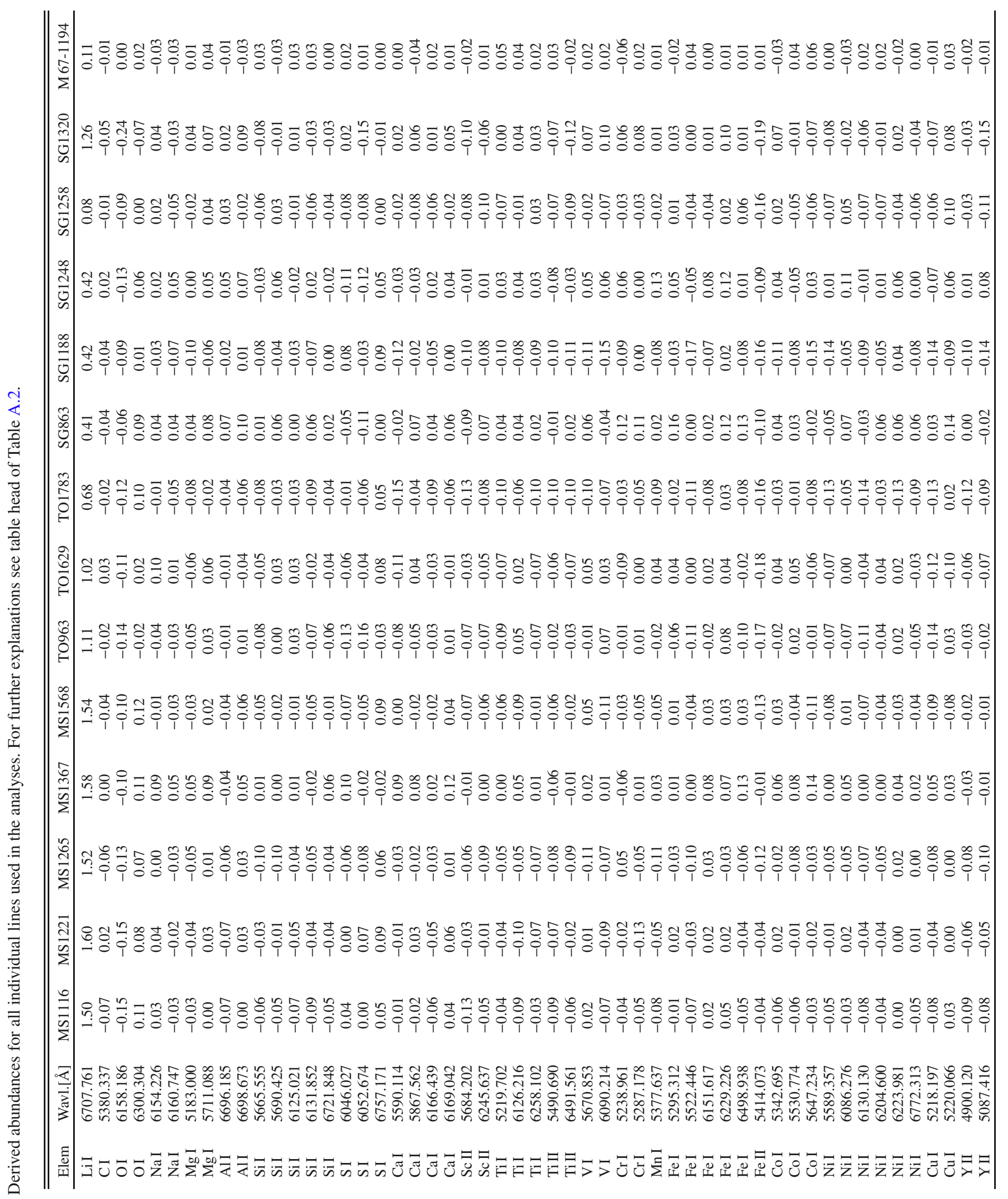




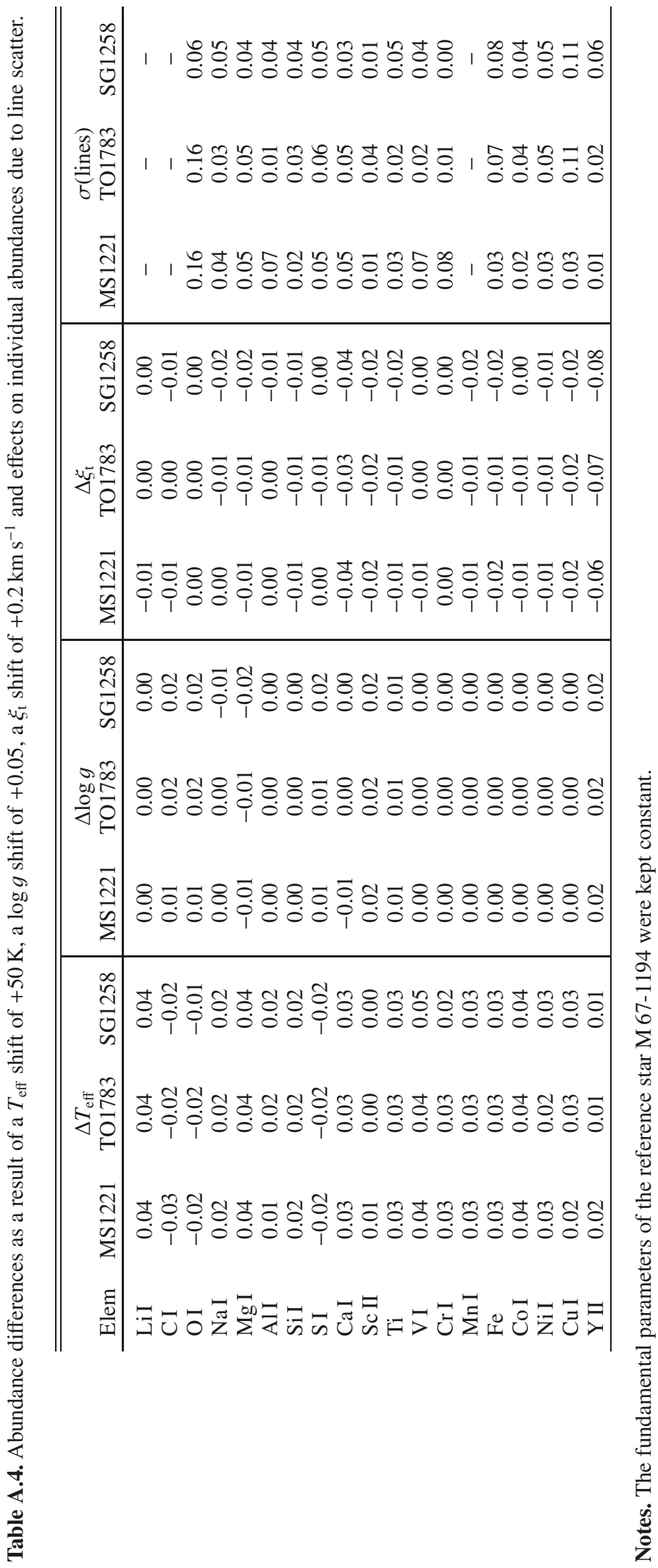

